\title{
Removal of Drops from Liquid-Liquid Dispersions Upon Flow Through Screens
}

\author{
C. G. VINSON* AND S. W. CHURCHILL† \\ Department of Chemical and Metallurgicul Engineering, \\ University of Michigan, Ann Arbor, Michigan (USA)
}

(Received: 17 November, 1969; in final form: 9 March 1970)

\begin{abstract}
Fibre-packed coalescers are widely employed to remove drops from liquid-liquid dispersions. This work has as its objective a quantitative correlation of drop removal with filtration variables. Photo-etched screens, which were positioned perpendicular to the flow, served as geometrically defined representations of fibrous media. Forward-angle light scattering was employed to measure the extent of drop removal for a variety of screen geometries, flow rates and physical properties of oil-in-water dispersions. The dispersions, which were prepared by homogenisation, had a narrow range of drop sizes whose average was approximately three microns. The measurements indicate that drop interception, screen geometry, viscous shear and dropfilament adhesion are the main factors which govern drop removal. The redispersion of the drop phase which had accumulated on the filaments was generally not observed. The data for the removal of uniform drops by screens with fixed surface energy were correlated with the product of interstitial velocity, continuous phase viscosity and the projected width of the filament in the plane of the screen.
\end{abstract}

\section{INTRODUCTION}

Liquid-liquid dispersions can often be clarified by passage through a fibrous mat. The mat imposes a tortuous path upon the drops. The probability that a drop will collide with a fibre is normally large in comparison with the probability that it will collide and coalesce with another dispersed drop in the

* Present address: Diamond Shamrock Corp., Box 348, Painesville, Ohio 44077, USA.

† Present address: School of Chemical Engineering, University of Pennsylvania, Philadelphia, Pennsylvania 19104, USA. flowing stream. If retained on collision, a drop can move along the scavenging fibre, move on to other fibres and unite with other retained drops. Retention depends either upon the physical entrapment of the drop or upon the ability of the drop to wet the fibres preferentially. The latter is normally required for suitable removal efficiency. In steady state operation, the volumetric rate of attachment of the dispersed phase to the fibres equals the volumetric rate of disengagement. Removal in the present context pertains to the steady state and signifies a reduction of the population of drops of a certain size.

There have been few previous attempts to characterise drop filtration by fibrous media quantitatively. Burtis and Kirkbride ${ }^{1}$ found that the extent of desalting of crude oil by Fiberglas increased with an increase in temperature and fiber density and a decrease in flow velocity. Voyutskii, et al. ${ }^{2}$ found that fibrous media became ineffective in removing water droplets from hydrocarbons at a sufficiently high velocity. They introduced the notion of optimum fibre wettability and attributed the appearance of secondary emulsions in the effluent to excess wetting by the dispersed phase. Rose and Kintner ${ }^{3}$ used light transmission to measure coalescence on a variety of fibrous media and found that the nature of the fibre had a pronounced influence.

Drop-filament collisions proceed by similar mechanisms for both aerosol collection and drop removal from liquid-liquid dispersions. Theories and experiments on aerosol collection are therefore relevant even though the Reynolds numbers are usually much larger. One of the most illuminating investigations as well as one of the first was by Witzmann ${ }^{4}$ who passed dye aerosols through fibrous glass papers and glass frit. Using light transmission and Brownian motion, he found that the filtration efficiency increased with an increase in the ratio of drop to pore size. The extent of fibre wettability by the drops appeared to be important.

The Chemical Engineering Journal (1) (1970)—@ Elsevier Publishing Company Ltd, England-Printed in Great Britain 
Special techniques are required in order to ascertain quantitatively the extent of drop removal by fibrous filters. Typical dispersions have a broad range of drop sizes; the fibres in a mat have a random spacing; and fibre wettability cannot be characterised under dynamic conditions and frequently changes during a single experiment. The objective of this work was to obtain a direct measure of drop concentrations and to fix the other variables such that the fraction of drops removed could be correlated with flow rate, filter geometry and the physical properties of the dispersions. Electrical and visco-elastic effects were excluded by the choice of particular liquid-liquid systems.

\section{EXPERIMENTAL WORK}

Photo-etched nickel screens, a tool of electron microscopy, were used as filter media. Their dimensioned interstices served as an idealised but geometrically defined representation of a fibre-mat filter. A discrete number of layers of screen were supported in a circular channel transverse to the stream of dispersion. The chemical nature of the surface of the screens was fixed by the addition of an absorbed wetting agent. Oil-in-water dispersions which contained about 0.05 volume percent of oil were prepared in a homogeniser. These dispersions had a narrow range of diameters and an average drop diameter between two and three microns. Forward-angle light scattering was used to perceive changes in the drop concentration and the size distribution upon the passage of the dispersion through the screens.

Figure 1 is a schematic diagram of the experimental equipment. The main item was the light-scattering detector. It was patterned after the one used by Chin, Sliepcevich and Tribus. ${ }^{5}$ Two collimated circular beams of monochromatic light at $546 \mathrm{~m} \mu$ were focused upon an aperture in front of a photomultiplier. The collimated beams were projected on thin channels of the dispersion flowing into and away from the screens. The angular distribution of intensity was obtained by moving the photomultiplier at constant speed across the cone of forward-scattered light.

The screens were die-cut to $\frac{1}{2}$-in diameter discs and were layered at a spacing of $\frac{1}{16}$-in in the filtration channel. $\Lambda$ maximum of five layers could be used. Since the screens were thin, each screen in the channel was supported by a thick planar 40 -mesh screen. The nickel screens, obtained from Buckbee Mears of St. Paul, and C. O. Jelliff of Southport, Connecticut, were fairly uniform as their projections show in Fig. 2. A fifth screen (not shown) was 400 mesh and had 26 per cent open area. The screens were prepared for preferential wetting by oil drops by immersion in a saturated solution of octadecylamine in heptane for several hours prior to a run.

The flow channels in the cells through which the dispersions were viewed had rectangular crosssections. The minor dimensions of the cross-sections

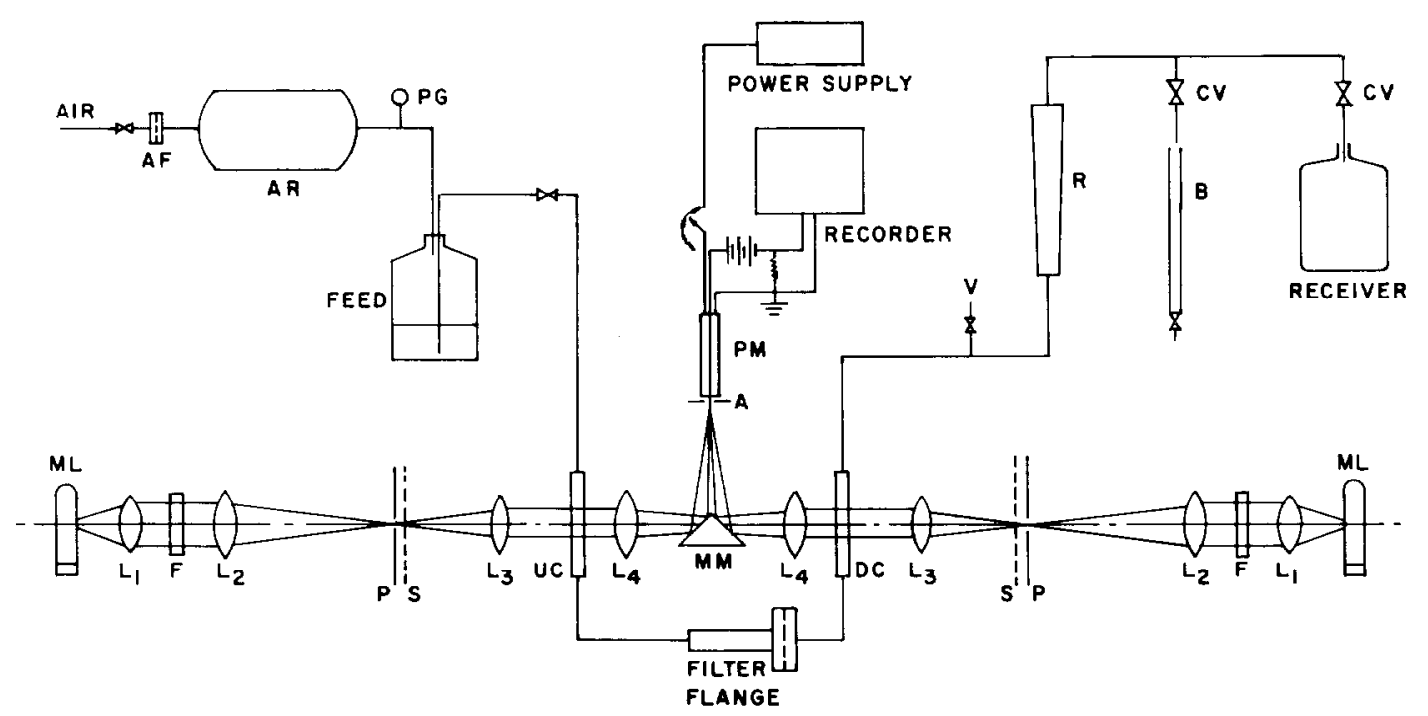

Fig. 1. Schematic diagram of experimental equipment.

$A$ - aperture, diam $=\frac{1}{8}$ in: $A F$ - air filter: $A R$ - air reservoir: $B$ - buret: $C V$-control valve: $F$-optical filter: $L_{1}, L_{2}, L_{3}, L_{4}$ - lenses: focal lengths $=79,343,176$ and $343 \mathrm{~mm}$, respectively: $M L$ - mercury lamp: MM - mirrors: $\mathbf{P}$ - pinhole, diam $=\frac{1}{16}$ in: $\mathbf{P G}$ - pressure gauge: $\mathbf{P M}$ - photomultiplier, Dumont 6291 : $\mathbf{R}$ - rotameter: $\mathbf{S}$-shutter: UC, DC - up-stream and downstream viewing cells: V - vent. 


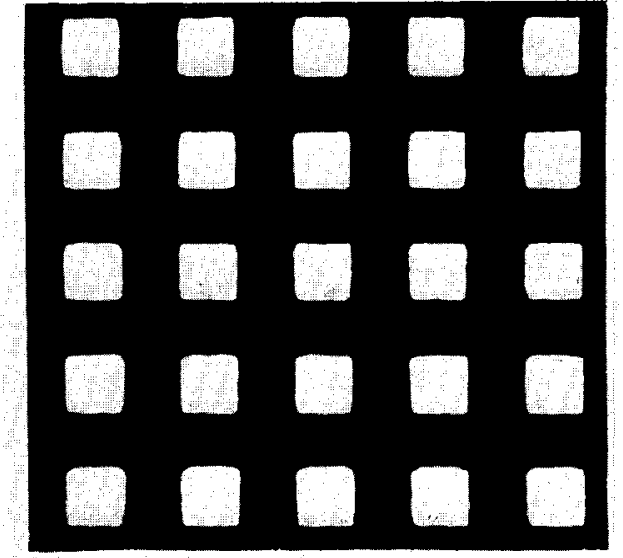

$500 \mathrm{MESH}, 25 \%$ OPEN AREA $M=1$
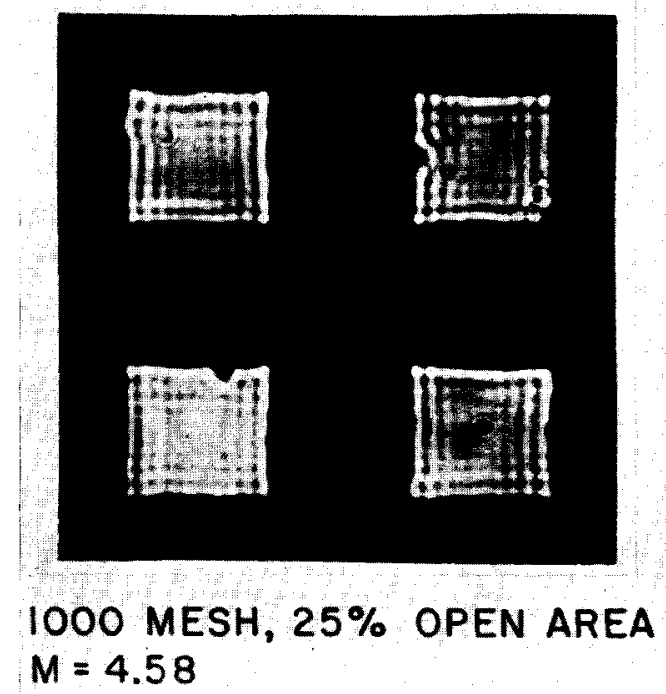

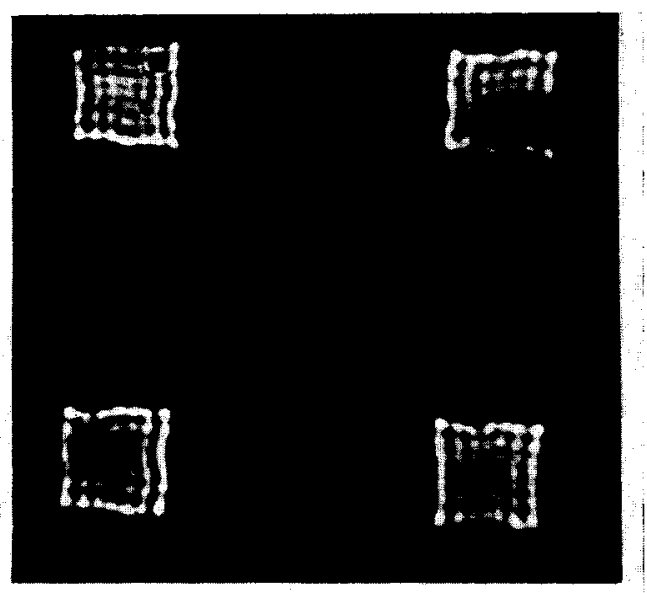

$750 \mathrm{MESH}, 9 \%$ OPEN AREA $M=4.58$

Fig. 2. Microphotographs of screens. $M=$ magnification relative to 500 mesh microphotograph.

were about 0.05 in and were parallel to the optical axes of the light scattering detector. The major dimensions of the cross-sections diverged gradually from the $\frac{3}{16}$-in inside diameter of the flow conduit to widths slightly larger than the $\frac{5}{8}$-in diameters of the collimated beams. Brass plate, microscope slides and epoxy resin were used in the construction of the viewing cells.

Cyclohexane-in-water dispersions were employed for most of the experimental work. Viscosities were incrcascd by adding glycerol and light mincral oil. Interfacial tension was reduced by using cyclohexanol and isobutanol. The drop-phase volume fraction was limited to 0.0005 in order to avoid multiple scattering.

Each experimental run was performed with a particular dispersion and set of screens. Companion scattering patterns were obtained in the viewing cells upstream and downstream from the screens at superficial filtration-channel velocities within the range of 1.5 to $120 \mathrm{~cm} / \mathrm{min}$. At each new rate of flow, the volume of fluid between the viewing cells was replaced one or more times before the scattering measurements were made. The flow was periodically surged in order to wash away accumulations of drop-phase in the flow channel. A run required up to three hours to completc.

\section{Light scattering calculations}

Chin, Sliepcevich and Tribus ${ }^{5}$ showed that the drop-size distribution can be determined directly and 
accurately from forward-angle scattering measurements with a lens-pinhole detector by utilising a Mellin transformation. This procedure is exact for Rayleigh diffraction. If the reduced diameter, defined as the ratio of the particle circumference to the wavelength, is greater than 50 , the scattering attenuates rapidly with increasing angle and the angular distribution given by the exact Mie theory does not differ appreciably from that given by Rayleigh diffraction. Moreover, the infinite transformation integral can be truncated at a low angle.

The reduced diameters for the drops and wavelength used in this investigation were in the range from 15 to 20 . Although these values could have been increased by using a shorter wavelength, other complications would have arisen. In order to test the assumption of diffraction theory for small reduced diameters a 2- to 3- $\mu \mathrm{m}$ fraction of Pyrex spheres was prepared. These were suspended in tetrabromoethylene and scattering patterns were obtained. A numerical approximation to the Mellin transformation was applied to the angular distribution of scattered intensity relative to the intensity of the transmitted beam. The Mellin integrand was computed for each integer multiple of $1 \mu \mathrm{m}$ for particle diameters between 1 and $12 \mu \mathrm{m}$ and for each integer multiple of 0.01 radian of polar angle for angles up to a maximum of 0.16 radian. Numerical integration with respect to angle at a fixed diameter gave a point value of the particlediameter distribution function. ${ }^{6}$ All point values which were negative were set to zero. In and near the range of diameters of the particles of the suspension, a series of positive point values were calculated for consecutive diameters. This was considered to be the diameter distribution. It was in effect bounded between two diameters near the known range of the suspended particles for which zero or negative point values were obtained via the integration. The diameter distribution was converted to a volume distribution by multiplying the point values by the corresponding diameter squared.

Figure 3 indicates a fair agreement between the distributions obtained by light scattering and those obtained from microscopic assay. The distributions calculated for the two compartments of the detector were nearly identical but were somewhat broader than those obtained by microscopic assay. The scattered intensity at all angles was found to be proportional to the concentration of the suspension. When applicd to a suspension of 4- to $11-\mu \mathrm{m}$ Pyrex spheres, the experimental procedure gave distributions which corresponded equally well with that obtained by microscopic assay. These comparisons were taken as evidence that the experimental and calculation procedure generates unique but slightly inexact distributions from the scattering of suspensions whose particles span a narrow range of diameters between 1 and $10 \mu \mathrm{m}$. It was observed that the maximum diameter in each suspension of Pyrex spheres was no more than three times the minimum. Since these restrictions were satisfied by the homogenised dispersions, the approximate calculation procedure was used for the analysis of the oilin-water dispersions.

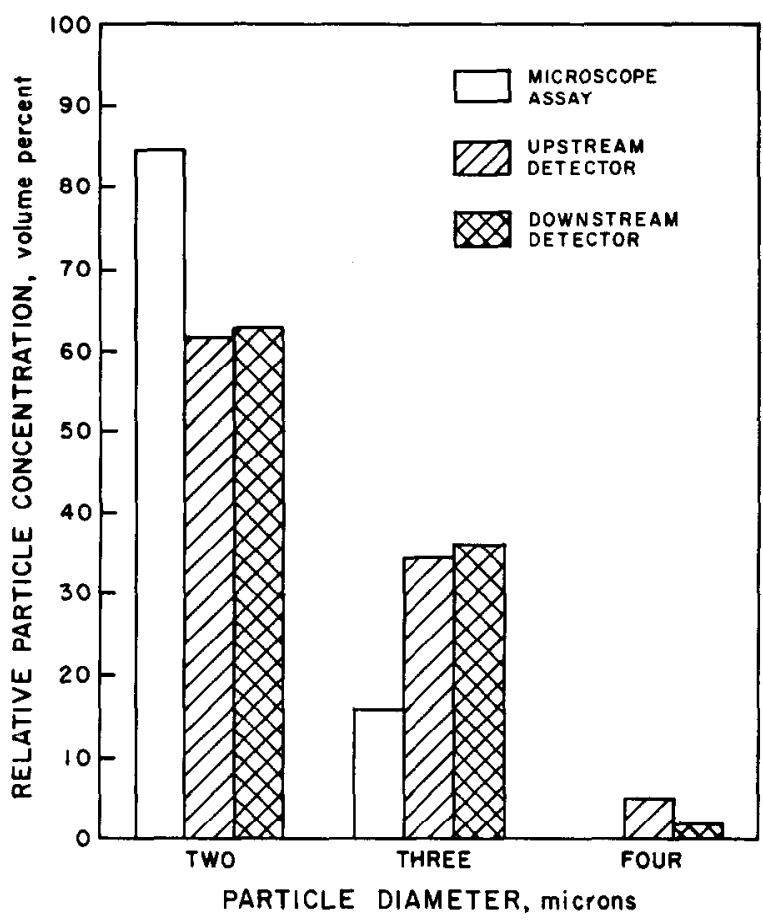

Fig. 3. Comparison of size distribution of suspension of Pyrex spheres as determined by microscopic assay and light scattering.

The error caused by the approximate integration was estimated by applying it to the hypothetical Rayleigh diffraction patterns for 2-, 3-, and 4- $\mu \mathrm{m}$ spheres. Figure 4 shows that the computed distributions peaked at a diameter somewhat smaller than that of the hypothetical value and tailed out to larger diameters. The distributions calculated from the scattering measurements of the filtration runs peaked at $2 \mu \mathrm{m}$. The distributions calculated at the beginning of a run were usually intermediate between the shapes calculated for the hypothetical diffraction patterns of 2- and 3- $\mu \mathrm{m}$ spheres. Toward the end of a run, the calculated distribution approached that of the hypothetical diffraction patterns for a $3-\mu \mathrm{m}$ sphere.

With the exception of the runs at elevated drop viscosity, the scattering patterns from the up- and downstream viewing cells were quite similar in shape 
at any point during a run. Since in theory the screens would be expected to discriminate between drop sizes, the near similarity suggests that the dispersions embraced a narrow range of drop sizes. Thus the extent of removal of drops of average size might have been estimated merely from the ratio of scattering intensities without recourse to the Mellin transformation. However, the detailed procedure was employed.
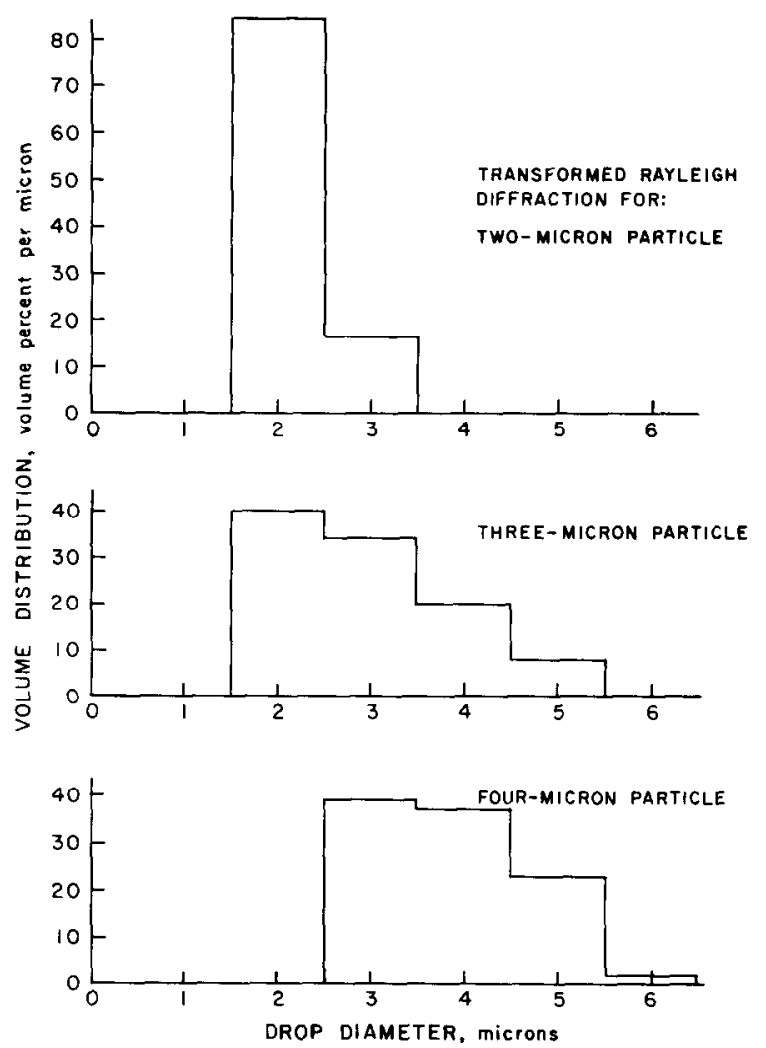

Fig. 4. Size distributions obtained from transformation of theoretical diffraction pattern of a single particle.

The fraction of drops not removed for a given set of conditions was calculated from the $2-\mu \mathrm{m}$ point value of the volume distribution of the downstream viewing cell ratioed to that of the upstream cell. Corrections were made for the difference in cell thickness and the extent of drop settling between cells. From the shapes of the volume distributions, it was inferred that the point value at $2 \mu \mathrm{m}$ most nearly represented $3-\mu \mathrm{m}$ drops. Drop removal data are reported as pertaining to such drops.

\section{ANALYSIS OF EXPERIMENTAL DATA}

Table 1 shows the range of variables covered in the runs performed. The Reynolds numbers based upon the interstitial velocity and the interstice width indicate that the flow was essentially laminar at the lowest velocities and that inertial effects may have been appreciable at the highest velocities. Four of the five different coalescing screens had equal interstice and filament dimensions. Neither density was varied appreciably. Since the density difference of the phases was between 0.15 and $0.35 \mathrm{gm} / \mathrm{cm}^{3}$ and since the drop diameter was approximately $3 \mu \mathrm{m}$, gravitational effects were insignificant. In the ambient temperature range of $16^{\circ}$ to $27^{\circ} \mathrm{C}$, viscosity was the only physical property whose variation with temperature had to be recognised.

TABLE 1

RANGE OF EXPERIMENTAL VARIABLES

\begin{tabular}{ll}
\hline Drop diameter, $\bar{d}_{P}$ & $3 \mu \mathrm{m}$, approximately \\
Filament width, $d_{F}$ & $8 \cdot 5-31 \cdot 4 \mu \mathrm{m}$ \\
Interstice width, $d_{I}$ & $8 \cdot 5-32 \cdot 1 \mu \mathrm{m}$ \\
$d_{F} / d_{I}$ & $0 \cdot 98-2 \cdot 46$ \\
Superficial velocity, $U_{\infty}$ & $1 \cdot 5-125 \mathrm{~cm} / \mathrm{min}$ \\
Interstitial velocity, $U_{0}$ & $12-1000 \mathrm{~cm} / \mathrm{min}$ \\
Continuous-phase viscosity, $\mu_{C}$ & $0 \cdot 9-9 \cdot 4 \mathrm{centipoise}$ \\
Drop-phase viscosity, $\mu_{D}$ & $0 \cdot 85-24 \cdot 0 \mathrm{centipoise}$ \\
Liquid-liquid interfacial tension, $\gamma_{C D}$ & $3-51 \mathrm{dyne} / \mathrm{cm}$ \\
Interstitial Reynolds number, $\rho_{C} U_{0} d_{I} / \mu_{C} 0.008-5 \cdot 1$
\end{tabular}

Screen wettability was not controlled in some of the runs but only those data for which the screens were treated with octadecylamine and for which flow rates were measured by timing the volume of control-valve effluent were used for correlation. The data obtained for interfacial tension less than 33 dyne $/ \mathrm{cm}$ and drop viscosity greater than $1 \mathrm{cp}$ did not satisfy these criteria.

The correlation pertains to the fractional removal of 3- $\mu \mathrm{m}$ drops by three equivalent layers of screen. In order that the data for five layers could be included, it was necessary to obtain experimental support for a rational adjustment from five layers to three.

If for certain conditions, each layer of screen removes the fraction $f_{1}$ of the drops incident to it, the fraction $f_{n}$ of the initial drops removed by $n$ layers of screen should be given by ${ }^{7}$

$$
f_{n}=1-\left(1-f_{1}\right)^{n}
$$

Equation (1) was examined for one to four layers of 1000 -mesh screen at various upstream velocities as indicated in Fig. 5. The scatter is believed to be primarily the result of flow measurement by a rotameter and secondarily to differences in screen wettability. Most of the data can be represented by straight lines which have the required intercept of 1.0 but intercepts for some of the higher velocities are above $1 \cdot 0$. Fractional removal was computed from a difference in concentrations. Relatively more 
error is present when the difference is small, as it tended to be at the higher velocities. These results were considered sufficient evidence to justify the application of eqn. (1) to convert the five-layer data to three layers.

Data for the fractional removal of $3-\mu \mathrm{m}$ drops by three equivalent layers of nickel screen treated with octadecylamine were correlated by the expression

$$
f_{3}=-0.089+0 \cdot 128\left(U_{o} d_{F} \mu_{C}\right)^{-0 \cdot 4}
$$

where the units of the independent variable are $(\mathrm{gm})$ (micron)/sec. ${ }^{2}$ The data used to develop the correlation are for interfacial tension $\geqq 33 \mathrm{dyne} / \mathrm{cm}$

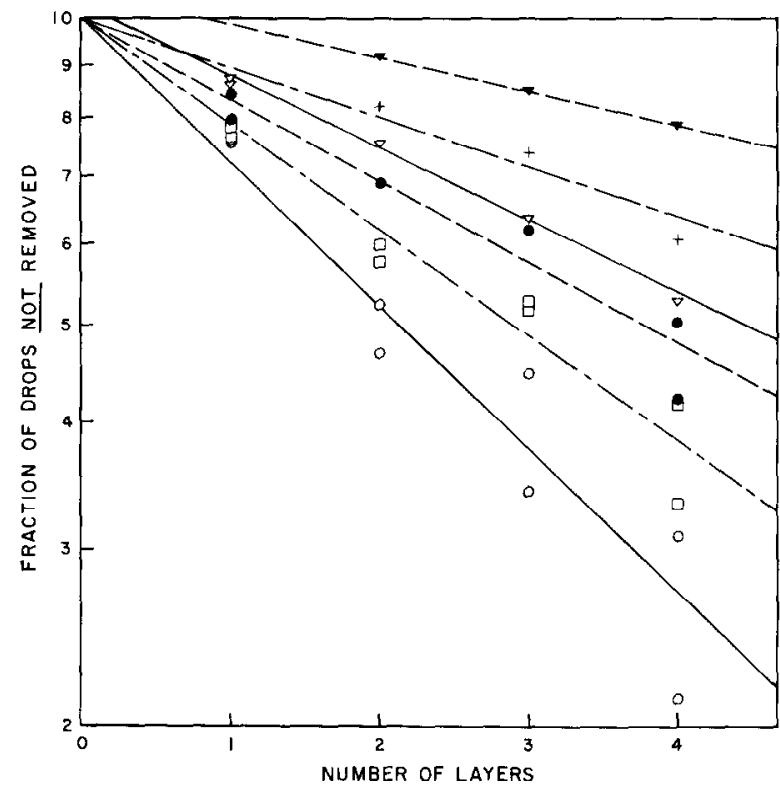

Fig. 5. Effect of number of layers of screen on drop removal. 1000 mesh screen. Symbols refer to superficial velocity,

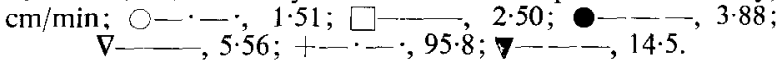

and drop viscosity $=1 \mathrm{cp}$. The fit of the data to eqn. (2) is illustrated by Fig. 6. Contrary to eqn. (2), fractional removal should probably approach zero asymptotically as $U_{o} d_{F} \mu_{C}$ increases. An inertial term would probably prove necessary at higher velocities.

The empirical constants and exponent in eqn. (2) have no significance other than for the experimental media. However, the same product of independent variables should be useful for correlating data for other situations of fractional drop removal by fibrous media.

Figure 7 indicates that coalescence dropped sharply as interfacial tension declined below 10 dynes $/ \mathrm{cm}$ but did not show much variation for larger values. The curves aid the visualisation but no

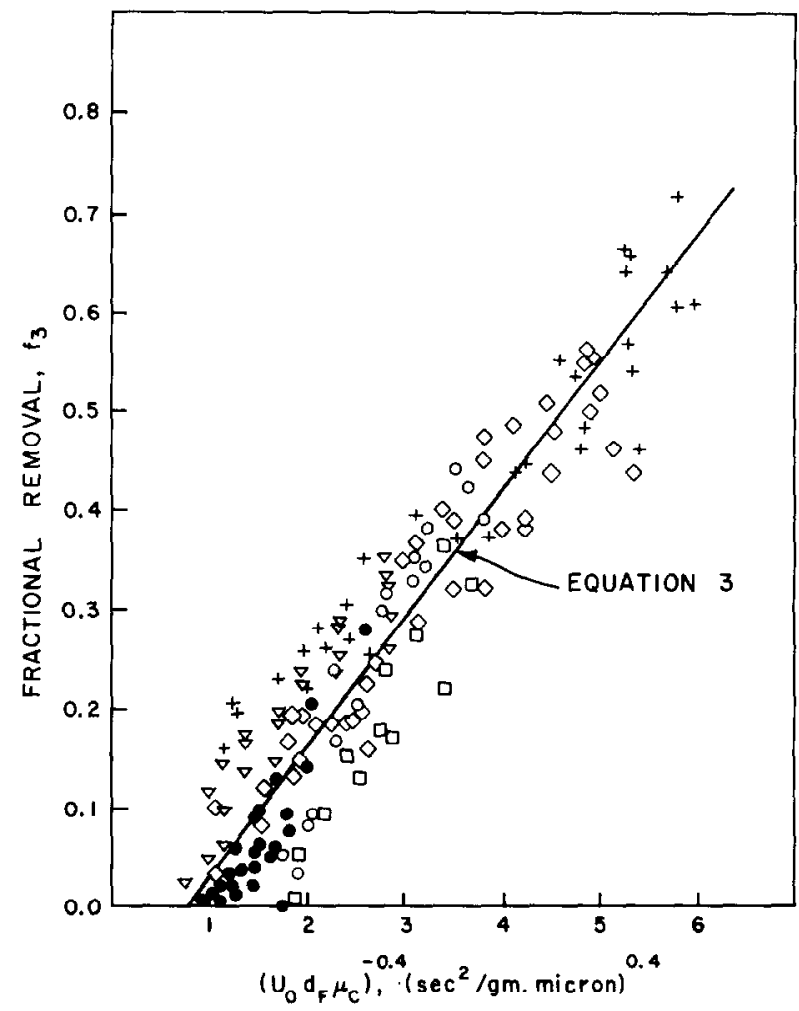

Fig. 6. Correlation of data. $\mu_{D}=1.0 \mathrm{cp} ; \gamma_{C D}=33 \mathrm{dyne} / \mathrm{cm}$; three equivalent layers of nickel screen treated with octadecylamine. Symbols refer to screen mesh. $\square, 400 ; \bigcirc, 500 ; \nabla, 750$; $\checkmark, 1000 ;+, 1500 ; 0,500$ with elevated continuous-phase viscosity.

significance is attributed to their shapes. The data for $11 \cdot 1$ dynes $/ \mathrm{cm}$ are for a fresh dispersion. Prolonged exposure of the screens to the systems of low interfacial tension caused a significant loss in coalescing ability. Hence it is suspected that the data for low interfacial tension show the effect of surface deterioration caused by amine desorption rather than a direct effect of interfacial tension. The data for $11 \cdot 1 \mathrm{dynes} / \mathrm{cm}$ suggest that coalescence at moderately low interfacial

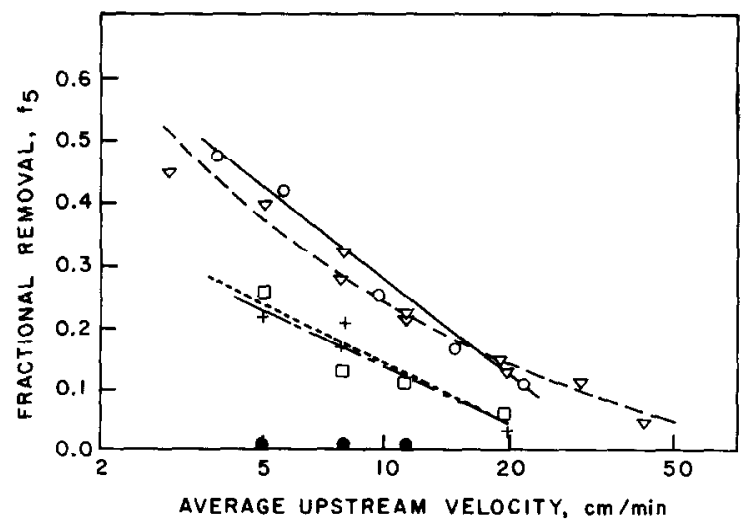

Fig. 7. Effect of interfacial tension on removal. Five layers of 500 mesh screen. Symbols refer to interfacial tension in dyne/ $\mathrm{cm} . \quad-51 ; \nabla-7 \cdot 7,11 \cdot 1 ; \square-\cdot-\cdot 7 \cdot 6 ;+\cdots, \cdot$ 
tensions is possible as long as screen wettability is maintained.

The data for dispersions of mineral oil in water reveal in Fig. 8 that coalescence increases slightly with drop viscosity. Most of the data show no definite trend in relation to the correlating line for the cyclohexane-in-water dispersion but there is a suggestion

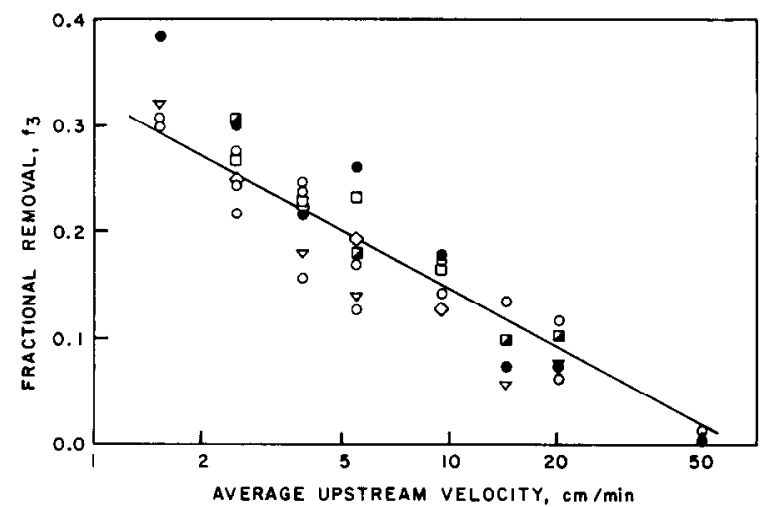

Fig. 8. Effect of drop viscosity on removal. Three layers of 1000 mesh screen. Symbols: $\bigcirc-, 0.88 \mathrm{cp} ; \nabla, 1.46 \mathrm{cp}$; $\square, 3 \cdot 0 \mathrm{cp} ;\langle, 5 \cdot 1 \mathrm{cp} ; \mathbf{\square}, 10 \cdot 1 \mathrm{cp} ; 0,24 \cdot 0 \mathrm{cp}$.

of increased coalescence at $24 \mathrm{cp}$. Runs at elevated drop viscosity did show a significant shift in the scattering patterns as the dispersion passed through the screens indicating that the proportion of small drops increased. The data in Fig. 8 indicale about half as much coalescence as the data in Fig. 6 because the screens were not saturated with respect to octadecylamine.

\section{GENERALISATIONS FROM EXPERIMENTAL DATA}

In the light of the present and previous investigations, drop capture on screen filaments embraces four possible governing phenomena: drop-filament juxtapositioning, the thinning of the continuous phase film between the drop and the filament, adhesive interaction between the drop and filament, and drop disengagement. Film rupture and the flow of the drop on to the filament are not included because they will almost invariably be fast. Drop motion on a filament and coalescence with another captured drop are also not considered likely to be rate controlling. Their occurrence depends upon the adhesive interaction.

The approach of a drop to the point of encounter with a filament may in the first approximation be treated according to the theories of particle interception which have been advanced for aerosols. ${ }^{8,9}$
Whenever the drop accelerates relative to the continuous phase, an additional account must be made of the drag of the drop on the surrounding fluid. ${ }^{10}$ Three- $\mu \mathrm{m}$ drops will not depart from a spherical shape except at negligible interfacial tensions. ${ }^{11}$

When a drop and a filament are in virtual contact, further motion of the drop toward the filament is retarded by the intervening film of continuous phase. The normal stresses required for film thinning can be generated by the flow and by van der Waals attraction. Contributions from Brownian motion will be negligible unless the drops are sub-micron.

When the film ruptures, the drop makes physical contact with the filament and flattens on to it. The extent of the flattening increases as the ability of the drop to wet the filament in the presence of the continuous phase increases. The flatter the drop, the less susceptible to fluid stresses. With good wettability, the filaments serve as low surface energy paths along which the flattened drop moves and unites with others.

The possibility that a drop will remain on the filament long enough to unite with others depends upon the drop-filament adhesion as well as wettability. Adhesion is viewed as a frictional resistance to the movement of the three-phase boundary

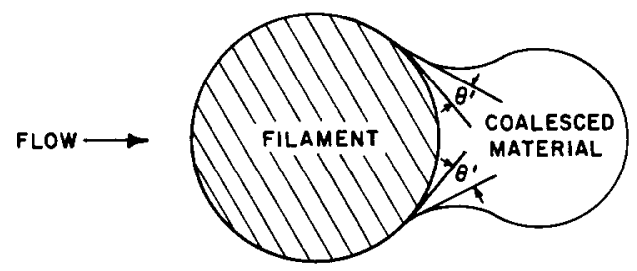

COALESCED MATERIAL CLINGING TO FILAMENT

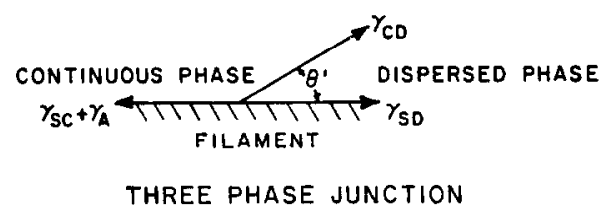

Fig. 9. Force balance at three-phase boundary.

between the two liquids and the filament. It arises from surface imperfections and absorption phenomena. ${ }^{12}$ Figure 9 illustrates the case of a globule of coalesced material which has been pushed to the downstream surface of a filament of circular crosssection. The indicated force balance at the dropfilament perimeter applies when fluid stresses have 
reduced the contact angle from its equilibrium value. In order for the perimeter to remain stationary, or for adhesion to prevail, the following inequality must be satisfied at all points.

$$
\gamma_{A}+\gamma_{S C}-\gamma_{S D}>\gamma_{C D} \cos \theta^{\prime}
$$

The larger the sum of the adhesive force, $\gamma_{A}$, and the wetting force, $\gamma_{S C}-\gamma_{S D}$, the larger the globule can grow before being dislodged. When adhesion is strong, cohesive failures may occur. Only a part of the globule would disengage. Cohesive failure $\bar{x}$ is the sole means of disengagement when the perimeter does not slip at a zero contact angle. This possibility depends upon either strong resisting forces or low liquid-liquid interfacial tension.

When cohesive failures prevail, the drop phase will be attenuated to sheets or threads downstream of the filaments by fiuid forces. The size of the drops disengaged depends upon the thickness of the sheets or threads, hence upon the amount of attenuation. The viscosity ratio, $\mu_{D} / \mu_{C}$, may affect the extent of attenuation in a manner similar to its effect on drop coalescence at a flat liquid-liquid interface. ${ }^{13}$ When $\mu_{D} / \mu_{C}$ is either large enough or small enough, coalescence occurs in a single stage. Apparently, a relatively large viscosity in one of the phases impedes the lateral contraction of the column formed by the drop as it drains into the liquid pool in the act of coalescence. Thus by retarding lateral disturbances on the interface, a large viscosity in one of the phases should allow more attenuation and the disengagement of smaller drops.

The phenomena of drop-filament approach, film thinning, adhesion and disengagement can be described by the seven independent dimensionless groups shown in Table 2. Groups describing film

TABLE 2

DIMENSIONLESS GROUPS WHICH CHARACTERISE DROP REMOVAL ON SCREENS

\begin{tabular}{ll} 
1. Drop interception & $\begin{array}{c}d_{P} / d_{F} \\
d_{P} / d_{I}\end{array}$ \\
3. Drop interception & $\left(\frac{\rho_{C} U_{0} d_{P}^{2}}{\mu_{C} d_{F}}\right)^{1 / 2}$ \\
4. Drop inertia & $\frac{A d_{F}}{U_{0} \mu_{C} d_{P}^{3}}$ \\
5. Adhesion & $\frac{\gamma^{*}}{\mu_{C} U_{0}}$ \\
6. Cohesion & $\gamma_{C D} / \gamma^{*}$ \\
7. Attenuation & $\mu_{D} / \mu_{C}$ \\
\hline
\end{tabular}

thinning by viscous and inertial forces are not independent of groups 1 and 3 . Adhesive and wetting forces, which cannot be measured, are combined in the variable $\gamma^{*}$. If the van der Waals attraction or the adhesion or cohesion groups were important, dimensional terms would necessarily be present in an empirical correlation of the data. Dimensional terms would also be present if other variables in the dimensionless groups were not varied. Equation (2) pertains to data for which $\rho_{C}, d_{P}, \gamma_{C D}, \gamma^{*}$ and $\mu_{D}$ were essentially fixed. Therefore, it is impossible to ascertain from the correlation whether the inertial, cohesion and attenuation groups are unimportant or whether the remaining groups are important. However, the form of the correlating group of variables and other observations are quite persuasive.

The inverse relation between fractional removal and the product $U_{0} d_{F} \mu_{C}$ points to the importance of drop interception and viscous shear. Data on the effect of drop size would be required to establish the role of interception but its importance is quite probable by the analogy with aerosol collection. The form of the independent variable is not suggestive of either inertial effects or film thinning. According to Fig. 7, removal was unaltered until the interfacial tension was less than $11 \mathrm{dyne} / \mathrm{cm}$. Before the octadecylamine treatment was adopted, fractional removal was inconsistent. Thus the combination of screen wettability and drop-filament adhesion are considered to be more important than interfacial tension. Interception, viscous shear and wettability and adhesion appear to be the most probable explanation for the other observed effects. They may be combined in the dimensionless product $\left(\gamma^{*} / U_{0} \mu_{C}\right)\left(d_{P} / d_{F}\right)$. It is not necessary for the exponents of the adhesion and interception terms to be the same but they both must be positive.

That $d_{1}$ did not appear explicitly in the correlation is probably the result of insufficient data on its independent effect. For screens with a low fractional open area such as those employed, $d_{p} / d_{l}$ would be expected to be a more important interception parameter than $d_{p} / d_{F}$. However $d_{l}$ cancels out when the product of the former interception parameter and the quotient of the characteristic adhesive and wetting stress over the interstitial shear stress is formed; i.e.,

$$
\left(d_{P} / d_{I}\right)\left(\gamma^{*} / d_{F}\right) /\left(\mu_{C} U_{o} / d_{l}\right)=\left(d_{P} / d_{F}\right)\left(\gamma^{*} / U_{o} \mu_{C}\right)
$$

'This cancellation requires equal exponents on the interception parameter and the stress ratio.

The data of Fig. 7 reveal that interfacial tension had an effect in the range of 10 dyne $/ \mathrm{cm}$ and less. However, it appears that the effect may have involved a change of adhesion and screen wettability as well as a promotion of disengagement by cohesive failure. At low interfacial tension, adhesion can be poor because of the inability of a surface to discriminate 
between liquids which are not totally alien in chemical properties. Figure 7 suggests that these effects are restricted to low levels of interfacial tension. It further appears from the undiminished initial coalescence at 11 dyne $/ \mathrm{cm}$ that the mechanisms indicated by the correlation continue to control in the presence of a moderate interfacial structure. They should therefore also be important for water-in-oil dispersions.

The runs at elevated drop viscosity were distinct because the screens caused a shift in the shape of the scattering patterns generated by the passing dispersions. The shift indicated that a larger proportion of small drops were present in the effluent. It is not certain that the greater attenuation, which might have originated with the elevated drop viscosity, was the cause. Disengagement by adhesive failure may have prevailed, since for these runs the screens were not treated and thus had a lower drop removal capacity. It is also possible that the screens removed the larger drops preferentially.

\section{CONCLUSIONS}

Light scattering measurements of the removal of drops from oil-in-water dispersions during flow past nickel screens indicate that removal is controlled by viscous shear, drop interception and the adhesion of the drop phase to the screen filaments. A statistical analysis of the drop removal data for slow flows, inviscid drops approximately $3 \mu \mathrm{m}$ in diameter, high interfacial tension and screens treated with octadecylamine indicate that the product $U_{0} d_{F} \mu_{C}$ is the best correlating variable. It is inferred that this variable will be significant in describing the removal of drops between 1 and $10 \mu \mathrm{m}$ in diameter on other filamental or fibrous media which are in a fixed state of wetting. Since a moderate interfacial structure does not seem to interfere as long as screen wettability is fixed, the correlating group should also be applicable to water-in-oil dispersions.

\section{NOMENCLATURE}

$A$ van der Waals constant, units of energy

$d_{F}$ projected width of filament in plane of screen

$d_{I}$ projected width of interstice

$d_{P}$ drop diameter

$f_{n}$ fraction of drops removed by $n$ successive layers of screen $n$ number of layers of screen

$U_{0}$ interstitial velocity; $U_{0}=U_{\infty}\left(1+d_{F} / d_{I}\right)^{2}$

$U_{\infty}$ superficial velocity in filter channel or average upstream velocity

\section{Greek symbols}

$\gamma_{A}$ lateral force of adhesion per unit length of threephase perimeter

$\gamma_{C D}$ interfacial tension between continuous and drop phases

$\gamma_{S C}$ interfacial tension between screen and continuous phase

$\gamma_{S D}$ interfacial tension between screen and drop phase

$\gamma^{*}$ sum of adhesive and wetting forces per unit length of three-phase perimeter

$\theta^{\prime}$ dynamic contact angle

$\mu_{C}$ continuous phase viscosity

$\mu_{D}$ drop-phase viscosity

$\rho_{C}$ density of continuous phase

$\mu \mathrm{m}$ micro metre

$\mathrm{m} \mu$ milli micro metre

\section{REFERENCES}

1. Burtis, T. A., and Kirkbride, C. G., Trans. A.I.Ch.E, 194642413.

2. Voyutskit, S. S., Kal Yanova, K. A., Panich, R. M., AND Fodiman, N. M., Doklady Akad. Nauk. SSSR, 1953 911155.

3. Rose, P. M., M. S. Thesis, Illinois Institute of Technology, Chicago, June 1963.

4. WitzmanN, H., Zeitschrift fur Elektrochemie, 194046343.

5. Chin, J. H., Sliepcevich, C. M., And Tribus, M., J. Phys. Chem. 195559841,845 .

6. Vinson, C. G., Ph. D. Thesis, University of Michigan, Ann Arbor, 1965.

7. Langmuir, I., O. S. R. D. Report No. 865 , Serial No. 335 , 1942.

8. Chen, C. Y., Chem. Reviews, 195555595.

9. FuCHs, N. A., Mechanics of Aerosols, Academy of Sciences, USSR, Moscow, 1955, pp. 68-74, $12329,240-58$; translation by U.S. Army Chemical Warfare Laboratories, Special Publication 4-12.

10. Hughs, R. R., ANd Gilliland, E. R., Chem. Eng. Progress, 195248497 .

11. Landau, L. D., AND Lifschitz, E. M., Fluid Mechanics, Pergamon Press, New York, 1959, p. 96.

12. TABOR, D., in EleY, D. D., Adhesion and Adhesives: Fundamentals and Practice, Society of Chemical Industry, London, 1954, p. 21.

13. Charles, G. E., and Mason, S. G., J. Colloid Sci., 1960 15105. 


\section{$R E ́ S U M E$}

Pour déplacer des gouttes à partir de dispersions liquide-liquide, on utilise fréquemment des coalescences du type lits fibreux. Le présent travail a pour but de relier quantitativement le déplacement des gouttes aux variables de le filtration.

Le milieu fibreux a été représenté par des écrans grillagés disposés perpendiculairement à la direction générale de l'écoulement. Le degré de déplacement des gouttes a été déterminé pour plusieurs géométries de grilles, diverses vitesses d'écoulement et propriétés physiques des dispersions d'huile dans l'eau, au moyen d'une méthode basée sur la dispersion de la lumière.

Les dispersions ont été préparées par homogénéisation; le diamètre moyen des gouttes était d'environ trois microns et la distribution des diamètres autour de cette moyenne était étroite.

Les mesures ont montré que les principaux facteurs gouvernant le déplacement des gouttes étaient les suivants: interception des gouttes, géométrie de la grille, tension visqueuse, adhésion des gouttes au filament. On n'a généralement pas observé la redispersion des gouttes à partir de la phase qui s'accumulait sur les filaments. Les valeurs obtenues dans le cas du déplacement de gouttes uniformes au moyen de grilles ayant une énergie superficielle donnée ont été reliées au produit de la vitesse intersticielle par la visocité de la phase continue et la largeur projetée du filament dans le plan de la grille.

\section{ZUSAMMENFASSUNG}

Mit Faserstoffen gefüllte Abscheider zur Entfernung von Tropfen aus Flüssig-flüssig-Dispersionen sind weit verbreitet. In dieser Arbeit werden die Beziehungen zwischen der Abscheidung und den Filtergrößen quantitativ untersucht. Senkrecht zur Strömung stehende, fotogeätzte Siebe dienten als geometrisch genau definierte Modelle der Faserstoffe. Die Vorwärtsstreuung des Lichtes wurde zur Messung der Abscheidung bei Variation der Siebgeometrie, des Durchsatzes und der physikalischen Eigenschaften der Dispersionen von öl in Wasser benutzt. Die durch Homogenisieren hergestellten Dispersionen hatten eine schmale Tropfengrößenverteilung, deren Mittelwert bei $3 \mu \mathrm{m}$ lag. Die Messungen zeigen, daß die mechanische Behinderung, die Siebabmessungen, die Scherspannung und die Haftkräfte zwischen Tropfen und Fasern die wesentlichen Einflußgrößen auf die Abscheidung sind. Eine erneute Dispersion der bereits abgeschiedenen Tropfen wurde im allgemeinen nicht beobachtet. Die Werte für die Abscheidung gleichartiger Tropfen durch Siebe mit einer bestimmten Oberflächenenergie ließen sich mit dem Produkt aus Geschwindigkeit in den Sieböffnungen, Zähigkeit der reinen Flüssigkeit und der Projektionsfläche der Siebstege korrelieren. 